\title{
Wertigkeit der MRT in der Diagnostik primärer und sekundärer zerebraler Infektionen
}

\author{
MR Imaging in the Diagnosis of Primary and Secondary Cerebral Infections
}

\author{
Autoren \\ K. Krope ${ }^{1}$, A. Speil ${ }^{2}$, G. Pantazis ${ }^{3}$, T. Nägele ${ }^{4}$, M. Horger $^{1}$ \\ Institute \\ Radiologie, Universität Tübingen \\ 2 Neurochirurgie, Universität Tübingen \\ 3 Neuropathologie, Universität Tübingen \\ ${ }^{4}$ Neuroradiologie, Universität Tübingen
}

\section{Key words \\ - brain \\ infection \\ - MRI}

eingereicht 31.7 .2012

akzeptiert 14.1.2013

Bibliografie

DOI http://dx.doi.org/

10.1055/s-0032-1330714

Online-Publikation: 14.3.2013

Fortschr Röntgenstr 2013; 185:

539-545 (c) Georg Thieme

Verlag KG Stuttgart · New York ·

ISSN 1438-9029

Korrespondenzadresse

Herr Dr. Kai Krope

Radiologie, Universität

Tübingen

Hoppe-Seyler Str. 3

72076 Tübingen

Tel.: $07071-2986510$

Fax: 0 7071-2986511

kai.krope@gmx.de

\section{Zusammenfassung}

$\nabla$

Zerebrale Infektionen können letal verlaufen, Voraussetzung für eine schnelle und effektive Therapie ist eine frühzeitige Diagnose. Eine allgemein anerkannte Methode ist die Bildgebung mittels MRT, in der vorliegenden Arbeit soll eine Übersicht über wichtige Sequenzen und charakteristische Bildmuster, jedoch auch über Grenzen ihrer Aussagekraft gegeben werden.

\section{Diagnose zerebraler Infektionen beim Immunkompetenten}

Bakterielle und mykotische Infektionen Bakterielle und mykotische Infektionen können sich als Abszesse manifestieren, letztere oft auch als Ischämien wegen des angioinvasiven Charakters der Infektion. Eine hämatogene Aussaat von Erregern insbesondere aus der Lunge ist bei mykotischen und bakteriellen Infektionen möglich. Bakterielle Infektionen können auch als Durchwanderungsabszess auftreten. Bei einem Trauma oder bei neurochirurgischen Eingriffen kann Fremdmaterial eindringen, häufigste Manifestationen einer Infektion sind neben dem Abszess eine Meningitis, seltener sind subdurale oder gar subperiostale Empyeme [1, 2]. Die klinische und laborchemische Diagnosestellung kann schwierig sein, da eine Leukozytenerhöhung den Anschluss an das Gefäßsystem voraussetzt, der durch eine Abszesskapsel verhindert werden kann. Eine Zellzahlvermehrung im Liquor tritt bei Anschluss an die Liquorräume auf, dann kann auch ein Pyozephalus resultieren. Die klinische Symptomatik ist variabel und reicht von Kopfschmerzen und Hirndruckzeichen über Krampfanfälle bis zur Bewusstlosigkeit. In vielen Fällen unverzichtbar ist die MRT zur Unterstützung der klinischen Diagnose.

\section{Abstract \\ $\nabla$}

Cerebral infections may develop into a life-threatening condition. Fast and correct diagnosis is crucial for a differentiated therapy and MRI imaging is widely accepted as the method of choice. Both specific MR sequences and imaging characteristics of major cerebral infections are addressed in this overview. Furthermore, limitations and pitfalls of the method are discussed.

Bildmorphologie bakterieller und mykotischer Abszesse

Die Entstehung von Abszessen ist an eine intakte Immunabwehr gekoppelt: Initialstadium ist eine lokale Zerebritis, im Rahmen der Immunantwort wird eine hochvaskularisierte Kapsel gebildet, die charakteristisch ist für einen reifen Abszess. Häufig sind Abszesse an der Mark-Rindengrenze lokalisiert und rundlich.

In der FLAIR findet sich ein hypointenser Randsaum. Ursachen für die Signalabsenkung sind die kollagenreichen Bestandteile der Kapsel, eine mögliche hämorrhagische Tinktion und, bei Immunkompetenz, die von Phagozyten freigesetzten freien Radikale. Der Randsaum ist in der nativen T1 isointens oder leicht hyperintens [3, 4]. Als indirektes Zeichen kann in der FLAIR ein Ödemsaum zu finden sein, der oft hauptverantwortlich für den raumfordernden Effekt ist.

Das Signal in der zentralen Abszesshöhle in der T1 und T2 ist stark von Proteingehalt und Viskosität abhängig. In der Diffusionswichtung (DWI) ist ein hohes Signal zu finden, der ADC ist verringert. Als physikalisches Korrelat der Diffusionseinschränkung kann die hohe Viskosität des Pus angesehen werden $[5,6]$. In einer Ex-vivo-Studie zeigten Mishra et al., dass die Dichte von Entzün- 

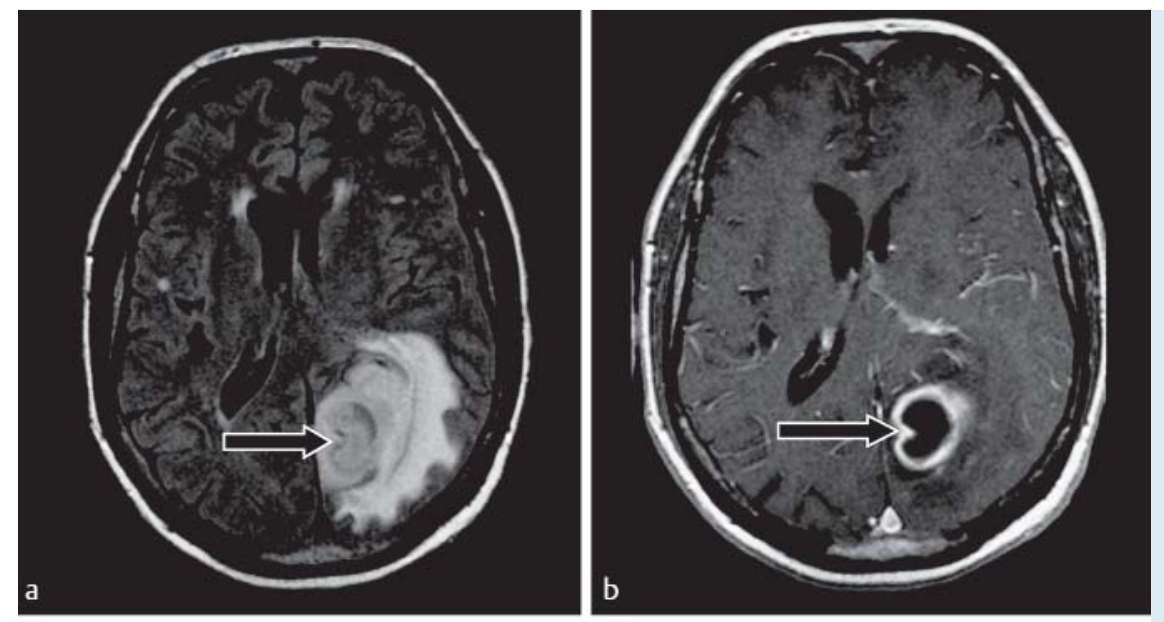

Abb. 1 Bakterieller, intraoperativ gesicherter Abszess. 54-jähriger Patient mit Hemianopsie nach rechts und Quadrantenanopsie nach links oben. In der FLAIR a lässt sich ein ausgedehnter, hyperintenser Ödemsaum im linken parietookzipitalen Marklager nachweisen sowie die hypointense Abszesskapsel (Pfeil). Nach Kontrastmittelgabe findet sich in der T1 ein ringförmiges Enhancement (Pfeil) der Läsion b. In der Diffusionswichtung c lässt sich eine Diffusionsstörung (Pfeil) mit ADC(Apparent Diffusion Coefficient)-Verminderung (Pfeil) $\mathbf{d}$ nachweisen.
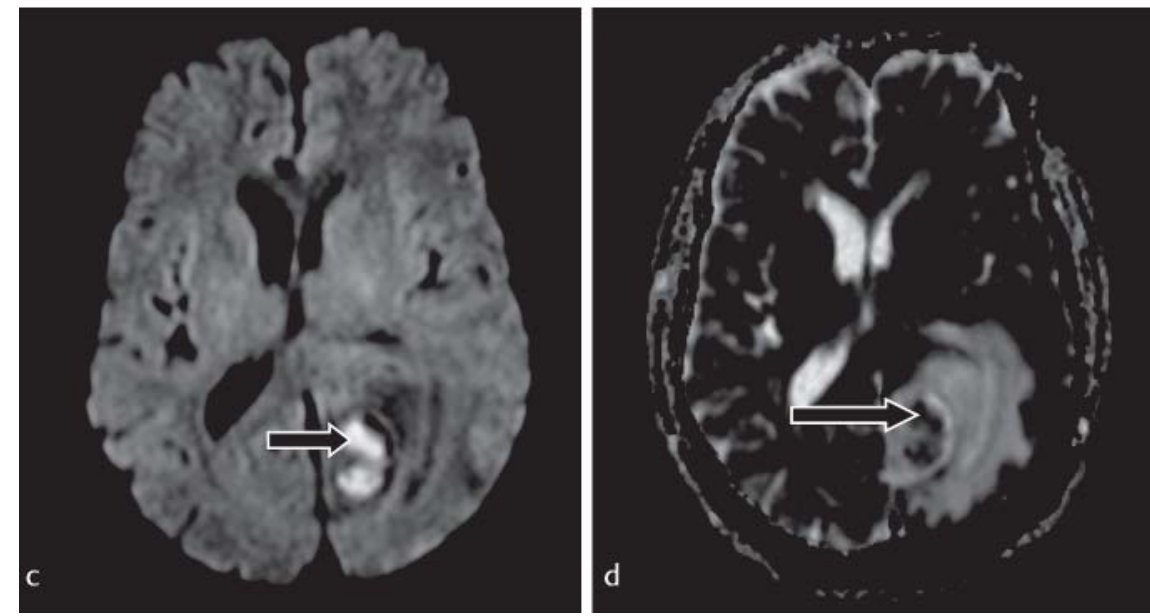

dungszellen den ADC-Wert beeinflusst [7]. $\bullet$ Abb. 1 zeigt Beispiele. Die Frage der Differenzierbarkeit von bakteriellen und mykotischen Abszessen ist Gegenstand vieler Studien. Luthra et al. [3] zeigten in ihrer Studie, in der die Signaleigenschaften von $\mathrm{n}=91$ bakteriellen und $\mathrm{n}=8$ mykotischen Abszessen untersucht wurden, dass eine Unterscheidung zwischen den Abszessen unterschiedlicher Genese möglich ist: Bei bakteriellen Abszessen ist eine Diffusionseinschränkung der zentralen Abszesshöhle nachweisbar, die sich bei mykotischen Abszessen nicht findet.

Limitationen in der Diagnose von Abszessen mittels DWI Wichtige Limitation der DWI ist die Differenzierung zwischen postoperativen Abszessen und hämatogenen. Während deren Erscheinungsbild sich in FLAIR und T1 ähnelt, lässt sich die für die Abszesshöhle typische Diffusionsstörung zwar bei hämatogenen Abszessen finden, bei postoperativen Abszessen kann sie fehlen. Wird nur die ADC zur Diagnose postoperativer Abszesse herangezogen, kann es zu falsch negativen Ergebnissen kommen mit einer Rate bis 36\% [8]. Als Ursache wird unter anderem die unterschiedliche Viskosität vermutet. Eine weitere Limitation betrifft sterile Abszesse: Mishra et al. [7] fanden in wenigen Fällen von sterilen Abszessen ein Fehlen der Diffusionseinschränkung, die aber auch bei positivem Erregernachweis fehlen kann.

\section{Differenzialdiagnose des zerebralen Abszesses mit mo- dernen Sequenzen}

Das Erscheinungsbild von Abszessen, Glioblastomen, Metastasen und Lymphomen ist in den konventionellen Sequenzen ähnlich, die Differenzierung kann schwierig sein. Die Informationen aus
DWI, Perfusion und DTI können weiterführen: Wang et al. [9] fanden, dass der kontrastmittelaffine Anteil von Glioblastomen eine höhere Anisotropie besitzt als der von Metastasen und Lymphomen. In der Perfusion konnten sie für Glioblastome und Metastasen einen höherer CBV(zerebrales Blutvolumen)-Wert nachweisen als für Lymphome. Viele nekrotische und zystische Hirntumoren haben ein intermediäres Signal in der DWI $[5,10]$. Relativ neu ist die SWI, mit der Blut und Kalzium differenziert werden können, die aber auch zur Beurteilung von Eisen geeignet ist. Mittels KM (KM-SWI) lassen sich Blutgefäße von Kalk und Blutablagerungen abgrenzen, letztere können durch Analyse der Phasenbilder differenziert werden [11]. Die KM-SWI kann zur Differenzierung zwischen Metastasen und Glioblastomen nützlich sein [12].

\section{Neurotuberkulose}

Erreger ist Mykobakterium tuberkulosis. Ort der Primärinfektion ist meist die Lunge, seltener Darm und Haut [13]. Die Infektion kann beim Immunkompetenten zunächst asymptomatisch verlaufen und reaktiviert werden. Extrapulmonale Manifestationen sind bei AIDS häufig, eine Neurotuberkulose ist mit einer hohen Mortalität behaftet. Die kulturelle Erregeranzucht kann 4 - 8 Wochen dauern, die Bildgebung kann eine frühe Diagnose ermöglichen: Häufig ist eine basal lokalisierte Meningitis, die von Vaskulitis und Infarkten begleitet sein kann. Exsudate in den basalen Zisternen können zu einem Hydrozephalus führen. Seltener sind Abszesse und Tuberkulome, letztere sind kleiner als Abszesse, können Verkalkungen oder Nekrosen aufweisen und in Parenchym, Meningen und Subarachnoidalraum lokalisiert sein. In 
der KM-T1 lässt sich ein ringförmiges Enhancement nachweisen $[10,14]$. Charakteristisch ist die Vergesellschaftung von Granulomen, basaler Meningitis, Hydrozephalus und Infarkten.

\section{Neuroborreliose}

Erreger der Lyme-Borreliose ist das Bakterium Borrelia burgdorferi, Überträger sind Zecken. Das Initialstadium ist gekennzeichnet durch grippeähnliche Symptome, nach einer Latenzzeit von 3-4 Wochen kann eine disseminierte Infektion auftreten, die vorrangig Nervensystem, Gelenke und Herz betrifft. Neurologische Symptomen sind bei Erwachsenen periphere Paresen, radikuläre Schmerzen und ein entzündliches Liquorsyndrom. In der Spätphase kann es nach Monaten oder Jahren zu Vaskulitis, Enzephalitis und Enzephalomyelitis kommen mit progredienten motorischen Störungen und Demenz. Eine ZNS-Beteiligung ist selten. Die Diagnose wird durch Nachweis von spezifischen intrathekalen Antikörpern gesichert.

Die Neuroborreliose kann dem Nachweis in der MRT entgehen. Das Erscheinungsmuster ist ein Mischbild: Es können Abszesse, bevorzugt in Kleinhirn und Hirnstamm, auftreten sowie T2-hyperintense, KM-affine Läsionen [15]. Bei einer Vaskulitis kommt dem Nachweis entzündlicher Gefäßwandveränderungen in der fettgesättigten KM-T1 besondere Bedeutung zu [16].

\section{Neurolues}

Erreger der Lues ist der Spirochät Treponema pallidum, die Übertragung erfolgt durch Sexualkontakte. Eine Koinfektion mit HIV ist häufig [17]. Es lassen sich 3 Krankheitsphasen unterscheiden, eine ZNS-Beteiligung ist häufig. Es können eine Meningitis, Hirnnervenneuritis und eine Polyradikulitis auftreten. Charakteristisch sind progressive Paralyse, Tabes dorsalis und meningovaskuläre Beteiligung mit Ausbildung von vaskulitischen Aneurysmen, Subarachnoidalblutungen und Infarkten. Psychiatrische Symptome sind Psychose und Demenz. Es lassen sich eine meningeale Verdickung nachweisen und unscharf berandete Gummen mit Ödem, die bevorzugte Lokalisation sind Zerebellum, Hirnstamm und Schädelbasis. In der T1 lässt sich ein Enhancement nachweisen, in der T2 ein hypo- oder isointenses Signal im Zentrum der Läsionen [15]. Auch eine Atrophie ist in vielen Fällen nachzuweisen [18].

\section{Prioneninfektionen}

Während die Diagnose einer wahrscheinlichen Creutzfeldt-Jakob-Krankheit (CJK) nach den Leitlinien der Deutschen Gesellschaft für Neurologie [16] klinisch gestellt und mittels Liquor- und MRT-Diagnostik unterstützt wird, erfolgt die endgültige Diagnose durch die Autopsie. Die MRT dient der Erhärtung der Verdachtsdiagnose.

Es lassen sich verschiedene Formen der CJK abgrenzen, die sporadische Form ist die häufigste Variante ( $\mathrm{sCJK}$ ). Die sCJK wird weiter unterteilt, bereits vor der molekularen Klassifikation wurden nach dem klinischem Erscheinungsbild verschiedene Varianten beschrieben (Heidenhain-, Oppenheimer-Brownell-, Stern-Garcin-Variante). Nach der molekularen Klassifikation lassen sich 6 Phänotypen unterscheiden, die neuropathologisch, klinisch und auch in der MRT differenzierbar sind [19]. Die Differenzialdiagnosen sind Demenzen, psychiatrische, entzündliche und maligne Erkrankungen. Bei alten Patienten kommen eine Alzheimererkrankung infrage, eine vaskuläre und eine Lewy-BodyDemenz [20], bei jungen eine chronische Enzephalitis [21]. Die Heidenhain-Variante ist klinisch gekennzeichnet durch das Auftreten visueller Störungen, eine Differenzialdiagnose sind unter anderem postiktale Veränderungen [22].

\section{Bildmorphologie der Prioneninfektionen}

Charakteristisch sind FLAIR-Signalanhebungen des Rindenbands und der Stammganglien, insbesondere des Thalamus. Hier lassen sich Diffusionsstörungen nachweisen. Die molekularen Subtypen lassen sich anhand unterschiedlicher Verteilungsmuster differenzieren, beispielsweise sind beim molekularen Subtyp VV2, der mit der Oppenheimer-Brownell-Variante gleichgesetzt werden kann, vorrangig die Stammganglien betroffen, während beim Subtyp MM2, der möglicherweise der Stern-Garcin-Variante entspricht, der Kortex häufiger Signalalterationen aufweist als die Stammganglien [23]. Die größte Sensitivität besitzt die DWI, auch die FLAIR ist geeignet, diese erfasst jedoch nicht immer die typischen Veränderungen [24, 25]. Insbesondere in der Frühphase der Erkrankung lassen sich in der FLAIR häufig keine Signalalterationen nachweisen, während sich in der mittleren Phase auch hier Veränderungen nachweisen lassen [24]. In • Abb. 2 werden Beispiele gezeigt.

Als neuropathologische Korrelate werden für die DWI spongiforme Veränderungen diskutiert, für die FLAIR die Gliose [19]. Schließlich lassen sich in der MR-Spektroskopie unspezifische metabolische Veränderungen nachweisen [26].

\section{Virale Infektionen}

Die häufigsten Verursacher von Virusinfektionen des ZNS beim Immunkompetenten sind Herpesviren (HHV) und Enteroviren
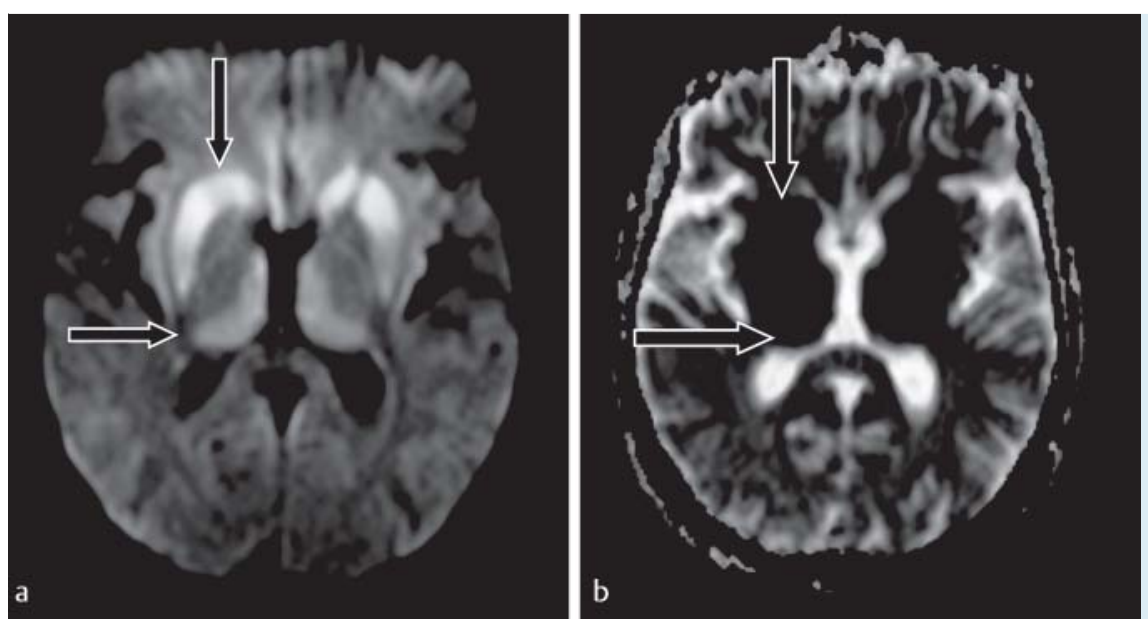

Abb. 2 Creutzfeldt-Jakob-Krankheit (CJK). 71-jährige Patientin mit rasch fortschreitender Demenz und Myokloni, im Verlauf trat ein akinetischer Mutismus auf. Postmortal wurde eine CJK gesichert. a In der Diffusionswichtung sind bihemisphärisch in Basalganglien und Thalamus Diffusionsstörungen nachzuweisen (Pfeile), korrespondierend hierzu findet sich in $\mathbf{b}$ eine Verminderung des ADC-Wertes (Pfeile). 


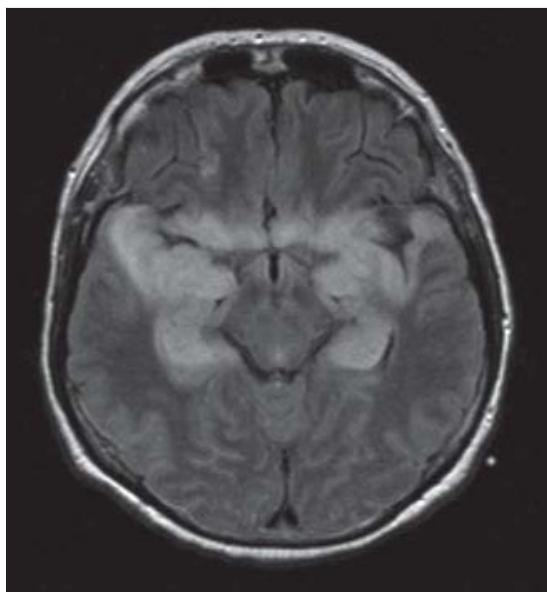

Abb. 3 HSV-1-Encephalitis. 66-jährige Patientin mit rezidivierenden generalisierten Krampfanfällen. In der FLAIR/T2 lassen sich Signalsteigerungen beider mesialer Temporallappen einschließlich der Hippocampusregion nachweisen, die das Rindenband mit einbeziehen. Das Sulkusrelief ist verplumpt.

[27]. Häufigste Infektion mit lebensbedrohlichem Ausgang ist die HSV-1-Enzephalitis, die daher im Folgenden näher vorgestellt wird. HHV-Infektionen können ein breites Spektrum neurologischer Symptome einschließlich Bewusstseinsstörungen, Hemiparese, und Krampfanfälle verursachen. Primäre Infektionen als Ursache einer Enzephalitis sind insbesondere bei Kindern möglich, bei Erwachsenen führt meist die Reaktivierung einer latenten Infektion zur Enzephalitis [28]. Da die neurologischen Symptome sich häufig rasch verschlechtern, ist auch bei dieser Infektion eine schnelle Diagnosestellung und Therapieeinleitung entscheidend [29]. Die wegweisende Untersuchung besteht in der Durchführung einer MRT [30]. Viruskulturen sind in weniger als $5 \%$ der Fälle einer HSV-1-Infektion positiv, diagnostischer Standard ist die PCR, deren Ergebnis jedoch erst nach 6-8 Stunden vorliegt [31].

Präsentation der Herpesenzephalitis ist die uni- oder bilaterale mesiotemporale T2- Signalsteigerung, als Ursache für dieses Verteilungsmuster, das oft eine leichte Seitenasymmetrie aufweist, kann die Ausbreitung des Virus entlang der meningealen Äste des N. trigeminus angesehen werden [32]. 0 Abb. 3 zeigt das Befallsmuster.

Das Erscheinungsbild bei Jugendlichen und Erwachsenen unterscheidet sich von den bei Kindern gefundenen Veränderungen [31]: Bei diesen finden sich häufig Hämorrhagien des medialen Temporallappens, des inferioren Frontallappens und der Inselregion, bei Neugeborenen sind diese selten [30]. Im frühen Krankheitsverlauf kann sich in der T1 ein normales Signal finden [30]. Bei der HSV-2-Infektion, die bei intrauterin infizierten Neugeborenen eine wichtige Rolle spielt, können außerdem Ischämien auftreten, häufig distant von den Primärmanifestationen lokalisiert [33].

Periolfaktorische Virusausbreitungen wurden ebenfalls berichtet [34]. Die Signalsteigerungen sind nicht - wie bei der CJD - auf das Rindenband begrenzt, die Entitäten lassen sich daher gut abgrenzen. Jedoch sind Einzelfälle berichtet, in denen beispielsweise eine Neurosyphilis [35] oder eine Infektion mit Listeria monocytogenes [36] mit einem solchen Verteilungsmuster einhergehen.

\section{Parasitosen}

Zystizerkose

Die häufigste Parasitose des Immunkompetenten ist die Zystizerkose, Erreger ist die Larve des Schweinebandwurms, Taenia solium. Die Übertragung erfolgt durch Lebensmittel. Eine Infektion führt in bis zu 90\% der Fälle zu einer ZNS-Beteiligung $[10,15]$.
Klinische Symptome sind Kopfschmerzen, Wesensänderung, epileptische Anfälle und fokal-neurologische Defizite.

Die Läsionen können an der Mark-Rindengrenze, im vierten Ventrikel und subarachnoidal lokalisiert sein. Bei Parenchymbefall lassen sich in Abhängigkeit des Stadiums verschiedene Erscheinungsbilder abgrenzen [15]:

1. Initialstadium: Es lässt sich nur ein fokales Ödem nachweisen, selten eine KM-Aufnahme. Es folgt die Ausbildung einer T1hypointensen, T2-hyperintensen Zyste mit einem Skolex (in $\mathrm{T} 1$ und $\mathrm{T} 2$ parenchymisointens).

2. Kolloidvesikelstadium: Der Proteingehalt der Zyste nimmt zu, das Signal ist T2-hyperintens. Um die Läsion wird eine fibröse Kapsel gebildet, es entsteht ein perifokales Ödem.

3. Granulär-noduläres Stadium: Die Läsion ist in T2 iso- oder hypointens, in der T1 isointens, es lässt sich eine noduläres oder ringförmiges Enhancement nachweisen. Das Ödem ist rückläufig.

4. Kalzifiziertes Stadium: Nach einer abgelaufenen Entzündung können sich punktförmige Verkalkungen nachweisen lassen.

Bei Liquorraumbefall komt es nicht zur Ausbildung eines Skolex. Die Zysten sind liquorisointens und nicht kontrastmittelaffin, jedoch motil.

\section{Echinokokkose}

Erreger sind Hunde- (Echinokokkus granulosus) bzw. Fuchsbandwurm (E. multilokularis). Die Infektion des Menschen erfolgt durch Ingestion, es erfolgt die hämatogene Aussaat mit Entwicklung von Hydatiden. Ein ZNS-Befall ist selten [37]. Symptome sind Liquorzirkulationsstörung, fokal-neurologische Ausfälle und epileptische Anfälle. Der Liquorbefund kann normal sein. Während die wandlosen Echinokokkus-multilokularis-Zysten invasiv wachsen und multiple Zysten aufweisen, ist das Wachstum der solitären Echinokokkus-granulosus-Zysten verdrängend. Die Zysten besitzen ein liquorisointenses Signal, eine Zystenruptur geht mit einer entzündlichen Umgebungsreaktion einher [15].

\section{Toxoplasmose}

Erreger der Toxoplasmose ist das Protozoon Toxoplasma gondii, die Übertragung kann in utero stattfinden [38], durch den Verzehr von rohem Fleisch, durch Kontakt mit Katzenkot oder durch Bluttransfusionen. Bei Immunkompromittierten sind mögliche Symptome Fieber, Wesensänderung, Krampfanfälle und das Auftreten fokal-neurologischer Defizite. Die Toxoplasmose ist die häufigste opportunistische Infektion bei manifester AIDS-Erkrankung [10]. Die Läsionen sind rundlich und nehmen exzentrisch Kontrastmittel auf. Das Signalverhalten florider Läsionen ist in der T2 variabel, in der nativen T1 sind die Läsionen isooder hypointens. Bevorzugte Lokalisation sind Basalganglien, Thalamus und Mark-Rindengrenze. Wichtigste Differenztialdiagnose sind Lymphome, die jedoch periventrikulär oder subependymal lokalisiert sind und ein isointenses Signal in der T2 aufweisen. Wichtige Zusatzinformationen liefern auch hier DWI, PWI und Spektroskopie [39]: Der ADC von Toxoplasmose-Herden ist höher als der ADC von Lymphomen. In der MR-Spektroskopie lassen sich in den Toxoplasmose-Läsionen hohe Lipid- und Laktat-Werte nachweisen, während andere Metabolite wie NAA fehlen. Hingegen lassen sich in Lymphomen Cholin- und NAA-Peaks nachweisen. Charakteristisch für Lymphome ist eine Hyperperfusion in der PWI [40]. 


\section{Wert der MRT in der Diagnose zerebraler Infektionen bei Immunkompromittierten \\ $\nabla$}

Beim Immunkompromittierten sind ZNS-Infektionen häufiger als beim Immunkompetenten, auch nehmen sie häufiger dramatische Verläufe. Die Ursachen einer Immunschwäche sind vielfältig und reichen von onkologischen und rheumatologischen Erkrankungen über Infektionen bis zur immunsuppressiven Therapie.

Das Erregerspektrum ist vielfältiger als beim Immunkompetenten und wird durch die opportunistischen Erreger erweitert, häufige Pathogene sind Toxoplasma gondii, Aspergillen, Kryptokokken, Kokzidiose und Kandida, jedoch auch die Reaktivierung einer Tuberkulose oder einer JC-Virusinfektion (siehe unten) ist möglich. Eine fast ausschließlich beim Immunkompromittierten auftretende Pilzinfektion ist die Mukormykose, der Erreger kann sich nach Inhalation von den Nasennebenhöhlen über Sinus cavernosus und Orbita bis ins Hirnparenchym ausbreiten [41]. Komplikationen sind beispielsweise Thrombosen der Arteria carotis interna und ein Hydrozephalus. Die Läsionen sind in der T1 hypointens, nehmen randlich Kontrastmittel auf, sind in der T2 hyperintens, besitzen raumfordernde Wirksamkeit und einen perifokalen Ödemsaum. Ein Beispiel ist in $\bullet$ Abb. 4 gezeigt.

Da eine adäquate Immunantwort nicht möglich ist, weichen häufig die typischen Bildmuster von denen beim Immunkompetenten ab: So fehlen häufig Abszesskapsel und Ödem. Findet die Ausbildung einer Abszesskapsel nicht statt, kann die Abgrenzung gegenüber einem Malignom erschwert sein. In diesem Fall kann die DWI eine Unterscheidung ermöglichen, da das Zentrum eines Abszesses in Abgrenzung zu einem Tumor meist eine Diffusionseinschränkung aufweist. Eine weitere Unterscheidungsmöglichkeit bietet die MR-Spektroskopie: Während sich in Abszessen Resonanzspektren bei 1,92 ppm (Azetat), 1,3 ppm (Laktat), 1,5 ppm (Alanin), 2,4 ppm (Sukzinat) und 0,9 ppm (Valin, Leuzin und Isoleuzin) nachgewiesen werden können, finden sich in Malignomen erhöhte Resonanzspektren von Cholin (3,2 ppm), Lipiden $(0,8$ und $1,5 \mathrm{ppm})$ und Laktat (1,3 ppm) mit Erniedrigung von $\operatorname{NAA}(2,0 \mathrm{ppm})$.

Häufigste Manifestation einer ZNS-Infektion ist die Meningitis [14]. In der FLAIR lässt sich oft eine Verdickung der Meningen nachweisen, in der T1 ein Enhancement. Der Post-KM-FLAIR wird die höchste Sensitivität zugesprochen.

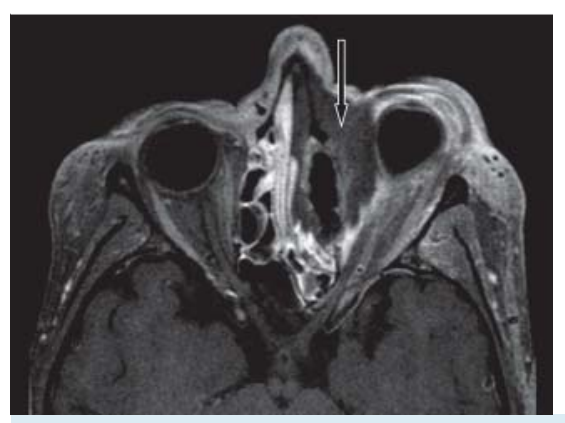

Abb. 4 Mucormykose. 42-jähriger Patient in Aplasie bei Rezidiv einer AML und einem seit einer Woche bestehenden Infekt der Nasennebenhöhlen. In der kontrastmittelangehobenen T1w-Nachweis einer zirkulären Weichteilverlegung des linken Sinus maxillaris (Pfeil), randlich KM-affin, zentral hypointens, die sich in die linke Orbita nach intrakonal fortsetzt, der Bulbus ist deformiert. Weitere Anteile der Raumforderung lassen sich im periorbitalen Weichteilmantel nachweisen. Die Dura ist am linken Temporalpol verdickt und nimmt Kontrastmittel auf.
Ist die Diagnose durch das Fehlen der typischen Bildmorphologie erschwert, ist der Stellenwert der MRT vermindert und der Erregernachweis aus Gewebeprobe, Blutkultur oder Liquor tritt in den Vordergrund.

Weil das Erscheinungsbild zerebraler Infektionen in der MRT beim Immunkompromittierten vielfältig ist, fokussiert sich die vorliegende Arbeit auf die Charakterisierung der Morphologie beim Immunkompetenten.

\section{JC-Virus und PML}

$\nabla$

Eine Infektion mit dem JC-Virus (JCV) kann sich als Progressive multifokale Leukenzephalopathie (PML) manifestieren [10, 42]. Nach der Primärinfektion, meist in der Kindheit, kann der JCV in renalen Tubuluszellen und lymphatischen Organen persistieren, eine Immunsuppression kann eine Reaktivierung der Infektion zur Folge haben. JCV-Antikörper können bei 86\% der Erwachsenen nachgewiesen werden [43]. Die Infektion tritt fast ausschließlich bei Immunkompromittierten auf und verläuft meist letal.

Die Infektion geht mit vielfältigen neurologischen und psychiatrischen Symptomen einher. Die Diagnose einer „sicheren PML“ wird durch histologische Sicherung oder Nachweis mittels PCR gestellt. Anhand einer MRT kann die Diagnose einer "möglichen PML" gestellt werden.

Es finden sich in der T1 Signalabsenkungen und in der T2 auffällig symmetrische Signalsteigerungen des parietalen, okzipitalen und frontalen Marklagers, die aufgrund des Verteilungsmusters einer toxischen Leukenzephalopathie ähneln können. $\mathbf{A}$ Abb. 5 zeigt ein Beispiel. Häufig ist auch eine symmetrische Verteilung im posterioren Marklager (DD posteriores reversibles Enzephalopatie-Syndrom). Die Beteiligung des Corpus callosum tritt meist zusammen mit der lobären Beteiligung auf, selten auch solitär, dann sind ein Lymphom und ein Astrozytom abzugrenzen. Selten sind Manifestationen in Pons und Mesenzephalon, atypisch ist die kortikale Beteiligung.

Anders als bei einer HSV-1-Infektion tritt eine Diffusionsstörung meist nicht auf. Ausnahmen sind Phasen aktiver Demyelinisierung und rascher Progredienz sowie Fälle einer begleitenden Entzündung [44]. Fehlen die typischen Läsionsmuster in den konventionellen Sequenzen, lassen sich in funktionellen Sequenzen Veränderungen nachweisen: In der MR- Spektroskopie lässt sich beispielsweise Cholin als Hinweis auf eine Demyelinisierung

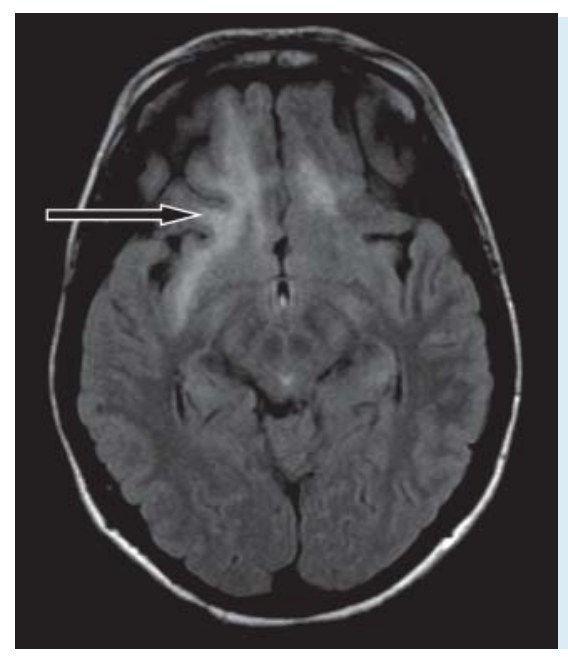

Abb. 5 Progressive multifokale Leukoenzephalopathie (PML). 23jährige Patientin mit JCV-Infektion bei HIV, generalisierte Krampfanfälle. In der FLAIR/T2 lassen sich Signalanhebungen im frontalen Marklager nachweisen, die seitenasymmetrisch mit Bevorzugung der rechten Hemisphäre ausgeprägt sind (Pfeil) und das Rindenband aussparen. 
nachweisen [39], in der DTI (diffusion tensor imaging) kann eine Verminderung der fraktionalen Anisotropie auch dann nachgewiesen werden, wenn Diffusionseinschränkungen nicht nachweisbar sind[45].

\section{JC-Virus: weitere Erkrankungen}

Neue Erkrankungen im Zusammenhang mit einer JCV-Infektion sind beschrieben [46, 47]: Befällt das JCV Neurone, kann es zu einer Granularzell-Neuronopathie (GCN) kommen. Manifestationsort ist das Zerebellum, Symptome sind Ataxie und Dysarthrie. Die Erkrankung kann isoliert oder zusammen mit einer PML auftreten. Die Diagnose wird durch eine Biopsie gesichert, in der MRT lässt sich eine zerebelläre Atrophie nachweisen.

Eine weitere Erkrankung ist die in einem Fallbericht beschriebene JCV-Enzephalitis [48]. Die Patientin hatte eine Chemotherapie erhalten und fiel durch eine rasche kognitive Verschlechterung auf. In der MRT waren zunächst eine Kortexbeteiligung, im Verlauf die Beteiligung subkortikaler Areale nachweisbar.

Mit einer JCV-Infektion kann auch eine Meningitis assoziiert sein, nicht sicher ist, ob es sich um eine Primärinfektion oder um eine Reaktivierung handelt. Nach der meist asymptomatischen Primärinfektion kann der Virus im ZNS persistieren. Symptome sind Nackensteifigkeit, Doppelbilder, Kopfschmerzen und Übelkeit. Die bei einer PML auftretenden fokalen Läsionen der weißen Substanz fehlen, es lässt sich eine geringe Erweiterung der inneren Liquorräume nachweisen. Der Virusnachweis im Liquor gelingt mittels PCR.

\section{IRIS und PML}

\section{$\nabla$}

Erholt sich das Immunsystem, kann ein IRIS (immune reconstitution inflammatory syndrome) mit neurologischer Verschlechterung auftreten, das mit einer hohen Mortalität behaftet ist. Eine Rekonstitution des Immunsystems tritt beispielsweise bei der Unterbrechung einer Natalizumab-Therapie bei einer PML und einer Enzephalomyelitis disseminata (ED) auf, aber auch bei HIV-Patienten unter antiretroviraler Therapie [49]. Drei verschiedene Verlaufsformen sind bekannt:

- die asymptomatische Form,

- die symptomatische Form mit subakuter Exazerbation vorbestehender PML-Symptome,

- die „katastrophale IRIS“ mit neurologischer Verschlechterung bis zu einem Koma.

Die mit der ED assoziierte IRIS scheint schwerere Verläufe zu nehmen als die mit einer HIV-Infektion assoziierte [50].

In der MRT können eine Größenprogredienz und (im Gegensatz zur „klassischen“ PML) ein vorübergehendes Kontrastmittelenhancement nachweisbar sein $[46,50]$.

\section{Zusammenfassung}

$\nabla$

Mittels MRT ist in vielen Fällen eine frühe Diagnose zerebraler Infektionen möglich. Beim Immunkompetenten lassen sich typische Erscheinungsbilder von mykotisch, bakteriell, viral, parasitär und durch Prionen bedingten Infektionen identifizieren. Die differenzialdiagnostische Abgrenzung von Läsionen mit ähnlichem Läsionsmuster - beispielsweise viraler und bakterieller Abszesse - ist mithilfe modernen Sequenzen möglich, aber auch die Unterscheidung beispielsweise zwischen Abszessen und nicht infektiös bedingten Läsionen.

Wichtige Limitationen der MRT bestehen bei der Anwendung der DWI zur Differenzierung von postoperativen Abszessen und bei der Diagnose zerebraler Infektionen beim Immunkompromittierten, bei dem das Erregerspektrum erheblich erweitert ist und die typischen Bildmuster fehlen können. In diesen Fällen tritt die histologische und serologische Diagnostik in den Vordergrund, der im Vergleich mit der MRT jedoch oft der Vorteil der frühzeitigen Diagnosestellung fehlt.

\section{Literatur}

1 McClelland S 3rd, Hall WA. Postoperative central nervous system infection: Incidence and associated factors in 2111 neurosurgical procedures. Clin Infect Dis 2007; 45: 55-59

2 Dashti SR, Baharvahdat $H$, Spetzler RF et al. Operative intracranial infection following craniotomy. Neurosurg Focus 2008; 24: E10

3 Luthra G, Parihar A, Nath $K$ et al. Comparative evaluation of fungal, tubercular, and pyogenic brain abscesses with conventional and diffusion MR imaging and proton MR spectroscopy. Am J Neuroradiol 2007; 28: $1332-1338$

4 Horger M, Nagele T, Joanoviciu SD et al. Bildgebung von Zerebritis und Hirnabszess. Fortschr Röntgenstr 2009; 181: 831 -835

5 Cartes-Zumelzu FW, Stavrou I, Castillo M et al. Diffusion-weighted imaging in the assessment of brain abscesses therapy. Am J Neuroradiol 2004; 25: $1310-1317$

6 Guzman R, Barth A, Lovblad KO et al. Use of diffusion-weighted magnetic resonance imaging in differentiating purulent brain processes from cystic brain tumors. J Neurosurg 2002; 97: 1101 - 1107

7 Mishra AM, Gupta RK, Saksena S et al. Biological correlates of diffusivity in brain abscess. Magnetic Resonance in Medicine 2005; 54: 878 - 885

8 Farrell CJ, Hoh BL, Pisculli ML et al. Limitations of diffusion-weighted imaging in the diagnosis of postoperative infections. Neurosurgery 2008; 62: 577-583; discussion 577-583

9 Wang S, Kim S, Chawla S et al. Differentiation between glioblastomas, solitary brain metastases, and primary cerebral lymphomas using diffusion tensor and dynamic susceptibility contrast-enhanced MR imaging. Am J Neuroradiol 2011; 32: 507-514

10 Karampekios S, Hesselink J. Cerebral infections. Eur Radiol 2005; 15 : $485-493$

11 Gasparotti R, Pinelli L, Liserre R. New MR sequences in daily practice: Susceptibility weighted imaging. A pictorial essay. Insights Imaging 2011; 2: 335-347

$12 \mathrm{Kim}$ HS, Jahng GH, Ryu CW et al. Added value and diagnostic performance of intratumoral susceptibility signals in the differential diagnosis of solitary enhancing brain lesions: Preliminary study. Am J Neuroradiol 2009; 30: 1574-1579

13 Heye T, Stoijkovic M, Kauczor HU et al. Extrapulmonale Tuberkulose: Die radiologische Bildgebung eines fast vergessenen Verwandlungskünstlers. Fortschr Röntgenstr 2011; 183: 1019-1029

14 Hartmann KM, Golinski M, Reith W. ZNS-Infektionen bei immunsupprimierten Patienten. Radiologe 2008; 48: 560-571

15 Hähnel S, Storch-Hagenlocher B, Kress B et al. Infektiöse Erkrankungen des Hirnparenchyms bei Erwachsenen: Bildgebung und differenzialdiagnostische Aspekte. Fortschr Röntgenstr 2005; 177: 1349-1365

16 Leitlinien für Diagnostik und Therapie in der Neurologie. 4. überarbeitete Aufl. Stuttgart: Georg Thieme Verlag; 2008

17 Ho EL, Lukehart SA. Syphilis: Using modern approaches to understand an old disease. J Clin Invest 2011; 121: 4584-4592

18 Peng F, Hu X, Zhong $X$ et al. CT and MR findings in hiv-negative neurosyphilis. Eur J Radiol 2008; 66: 1-6

19 Krope K, Pantazis G, Nagele $T$ et al. Sporadische Creutzfeld- JacobKrankheit. Fortschr Röntgenstr 2011; 183: 593-596

20 Haïk S, Brandel JP, Sazdovitch V et al. Dementia with lewy bodies in a neuropathologic series of suspected Creutzfeldt-Jakob disease. Neurology 2000; 55: 1401 - 1404

21 Poser S, Mollenhauer B, Kraubeta A et al. How to improve the clinical diagnosis of Creutzfeldt-Jakob disease. Brain 1999; 122: 2345-2351

22 Arruda WO, Bordignon KC, Milano JB et al. Doença de Creutzfeldt-Jakob forma heidenhain: Relato de caso com achados de ressonância magnética e dwi. Arq Neuropsiquiatr 2004; 62: 347-352 
23 Meissner B, Kallenberg K, Sanchez-Juan $P$ et al. MRI lesion profiles in sporadic Creutzfeldt-Jakob disease. Neurology 2009; 72: 1994-2001

24 Meissner B, Kallenberg K, Sanchez-Juan P et al. Isolated cortical signal increase on MR imaging as a frequent lesion pattern in sporadic Creutzfeldt-Jakob disease. Am J Neuroradiol 2008; 29: 1519-1524

25 Kallenberg K, Schulz-Schaeffer WJ, Jastrow U et al. Creutzfeldt-Jakob disease: Comparative analysis of MR imaging sequences. Am J Neuroradiol 2006; 27: 1459-1462

26 Galanaud D, Haik S, Linguraru MG et al. Combined diffusion imaging and MR spectroscopy in the diagnosis of human prion diseases. Am J Neuroradiol 2010; 31: $1311-1318$

27 Frantzidou F, Kamaria F, Dumaidi K et al. Aseptic meningitis and encephalitis because of herpesviruses and enteroviruses in an immunocompetent adult population. Eur J Neurol 2008; 15: 995-997

28 Johnson $R$. Viral infections of the nervous system. 2 ed. Philadelphia, New York: Lippincott-Raven; 1998

29 Whitley $R$. Herpes simplex virus. Philadelphia, New York: LippincottRaven; 1997

30 Leonard JR, Moran CJ, Cross DT 3rd et al. MR imaging of herpes simplex type 1 encephalitis in infants and young children: A separate pattern of findings. Am J Roentgenol 2000; 174: 1651 - 1655

31 Lakeman FD, Whitley RJ. Diagnosis of herpes simplex encephalitis: Application of polymerase chain reaction to cerebrospinal fluid from brain-biopsied patients and correlation with disease. National institute of allergy and infectious diseases collaborative antiviral study group. J Infect Dis 1995; 171: 857-863

32 Johnson B. Intracranial infections. Philadelphia: Saunders; 2000

33 Vossough A, Zimmerman R, Bilaniuk $L$ et al. Imaging findings of neonatal herpes simplex virus type 2 encephalitis. Neuroradiology 2008; 50 (4): $355-366$

34 Mori I, Nishiyama Y, Yokochi T et al. Olfactory transmission of neurotropic viruses. J Neurovirol 2005; 11: 129-137

35 Bash S, Hathout GM, Cohen S. Mesiotemporal t2-weighted hyperintensity: Neurosyphilis mimicking herpes encephalitis. American Journal of Neuroradiology 2001; 22: 314-316

36 Cunha BA, Fatehpuria R, Eisenstein LE. Listeria monocytogenes encephalitis mimicking herpes simplex virus encephalitis: The differential diagnostic importance of cerebrospinal fluid lactic acid levels. Heart Lung 2007; 36: $226-231$
37 Chang KH, Han MH. MRI of CNS parasitic diseases. Journal of Magnetic Resonance Imaging 1998; 8: 297-307

38 Bohlsen D, Vollmann R, Simbrunner J. Atypische zerebrale Toxoplasmose nach Nierentransplantation. Fortschr Röntgenstr 2011; 183: 762 - 764

39 Chang L, Ernst T. MR spectroscopy and diffusion-weighted MR imaging in focal brain lesions in aids. Neuroimaging Clin N Am 1997; 7: 409-426

40 Ernst TM, Chang L, Witt MD et al. Cerebral toxoplasmosis and lymphoma in aids: Perfusion MR imaging experience in 13 patients. Radiology 1998; 208: 663-669

41 Rapidis $A D$. Orbitomaxillary mucormycosis (zygomycosis) and the surgical approach to treatment: Perspectives from a maxillofacial surgeon. Clin Microbiol Infect 2009; 15: $98-102$

42 Horger $M$, Beschorner R, Beck R et al. Common and uncommon imaging findings in progressive multifocal leukoencephalopathy (pml) with differential diagnostic considerations. Clin Neurol Neurosurg 2012, Oct; 114 (8): $1123-1130$

43 Weber T, Trebst C, Frye S et al. Analysis of the systemic and intrathecal humoral immune response in progressive multifocal leukoencephalopathy. J Infect Dis 1997; 176: 250-254

44 Mader I, Herrlinger $U$, Klose $U$ et al. Progressive multifocal leukoencephalopathy: Analysis of lesion development with diffusion-weighted MRI. Neuroradiology 2003; 45: 717-721

45 Atlas SW. Magnetic resonance imaging of the brain. 4th ed. Philadelphia, Lippincott: Williams\&Wilkins; 2009

46 Tan CS, Koralnik IJ. Progressive multifocal leukoencephalopathy and other disorders caused by jc virus: Clinical features and pathogenesis. Lancet Neurol 2010; 9: 425-437

47 Behzad-Behbahani A, Klapper PE, Vallely PJ et al. Bkv-DNA and jcv-DNA in csf of patients with suspected meningitis or encephalitis. Infection 2003; 31: $374-378$

48 Wüthrich C, Dang X, Westmoreland S et al. Fulminant jc virus encephalopathy with productive infection of cortical pyramidal neurons. Ann Neurol 2009; 65: 742 - 748

49 Sahraian MA, Radue EW, Eshaghi A et al. Progressive multifocal leukoencephalopathy: A review of the neuroimaging features and differential diagnosis. Eur J Neurol 2012; 19: 1060 - 1069

50 Clifford DB, De Luca A, Simpson DM et al. Natalizumab-associated progressive multifocal leukoencephalopathy in patients with multiple sclerosis: Lessons from 28 cases. Lancet Neurol 2010; 9: 438-446 